JOURNAL OF

FUNCTION SPACES AND APPLICATIONS

Volume 4, Number 3 (2006), 261-273
(C) 2006, Scientific Horizon http://www.jfsa.net

\title{
The boundedness of multilinear commutators on locally compact Vilenkin groups
}

\author{
Canqin Tang \\ (Communicated by Hans Triebel)
}

2000 Mathematics Subject Classification. 43A70, 43A75.

Keywords and phrases. Multilinear commutator, Hardy space, Vilenkin group.

Abstract. Let $G$ be a locally compact Vilenkin group. In this paper, the authors investigate the boundedness of multilinear commutators of fractional integral operator on Lebesgue spaces on $G$. Furthermore, the boundedness on Hardy spaces are also obtained in this paper.

\section{Introduction}

The commutators have been studied by many authors for a long time. A well known result which is discovered by Coifman, Rocherg and Weiss ([3], [6], [9]) is that the commutators $[b, T]$ of singular integral operators are bounded on some $L^{p}\left(\mathbb{R}^{n}\right)(1<p<\infty)$ if and only if $b \in B M O$, where $[b, T]$ is defined by

$$
[b, T] f(x)=b(x) T f(x)-T(b f)(x) .
$$


A natural generalization of the commutator $T_{b}^{m}$ is given by

$$
T_{b}^{m}(f)(x)=\int_{\mathbb{R}^{n}}[b(x)-b(y)]^{m} K(x, y) f(y) d y,
$$

where $m \in \mathbb{N}$. It was shown in [1] that it is bounded on $L^{p}(\omega)(1<p<\infty)$ when $\omega \in A_{p}$. And in [2], the authors prove the $L^{p}(\omega)(1<p<\infty)$ boundedness for multilinear commutators. And similar results can be found in [10]. This is the motivation of considering the boundedness for multilinear commutators of fractional integral operator on locally compact Vilenkin group $G$.

In order to state our results more precisely we first introduce some notations and definitions.

Throughout this paper, $G$ will denote a bounded locally compact Vilenkin group, that is, $G$ is a locally compact Abelian group containing a strictly decreasing sequence of compact open subgroups $\left\{G_{n}\right\}_{n=-\infty}^{\infty}$ such that

(a) $\cup_{n=-\infty}^{\infty} G_{n}=G$ and $\cap_{n=-\infty}^{\infty} G_{n}=\{0\}$;

(b) $\sup \left\{\right.$ order $\left.\left(G_{n} / G_{n+1}\right): n \in \mathbb{Z}\right\}=B<\infty$.

Choose Haar measure $d x$ on $G$ so that $\left|G_{0}\right|=1$, where $|A|$ denotes the measure of a measurable subset $A$ of $G$. Let $\left|G_{n}\right|=\left(m_{n}\right)^{-1}$ for each $n \in \mathbb{Z}$. Since $2 m_{n} \leq m_{n+1} \leq B m_{n}$, it follows that

$$
\sum_{n=k}^{\infty}\left(m_{n}\right)^{-\alpha} \leq c\left(m_{k}\right)^{-\alpha}
$$

and

$$
\sum_{n=-\infty}^{k}\left(m_{n}\right)^{\alpha} \leq c\left(m_{k}\right)^{\alpha}
$$

for any $\alpha>0, k \in \mathbb{Z}$, where $c$ is a constant independent of $k$. For each $n \in \mathbb{Z}$ we choose elements $z_{l, n} \in G\left(l \in \mathbb{Z}_{+}\right)$so that the subsets $G_{l, n}:=z_{l, n}+G_{n}$ of $G$ satisfy $G_{k, n} \cap G_{l, n}=\phi$ if $k \neq l$ and $\cup_{l=0}^{\infty} G_{l, n}=G$; moreover, we choose $z_{0, n}$ such that $G_{0, n}=G_{n}$. We now define the function $d: G \times G \rightarrow \mathbb{R}$ by $d(x, y)=0$ if $x-y=0$ and $d(x, y)=\left(m_{n}\right)^{-1}$ if $x-y \in G_{n} \backslash G_{n+1}$. Then $d$ is a metric on $G$ and the topology on $G$ generated by this metric is the same as the original topology on $G$. For $x \in G$, set $|x|=d(x, 0)$. Then $|x|=\left(m_{n}\right)^{-1}$ if and only if $x \in G_{n} \backslash G_{n+1}$. Let $S(G)$ be the space of test functions and $S^{\prime}(G)$ be the distribution space on $G$. And $\chi_{G_{n}}$ is the characteristic function of $G_{n} . C$ can be denote various constants.

We also recall the definition of space of bounded mean oscillation. For $x_{0} \in G$, set $I_{j}=x_{0}+G_{j}$, we say a locally integrable function $b$ has bounded 
mean oscillation, $b \in B M O(G)$, if

$$
\sup _{I_{j} \ni x} \inf _{c \in \mathbb{R}}\left(\frac{1}{\left|I_{j}\right|} \int_{I_{j}}|f(y)-c| d y\right) \leq \infty
$$

where the supremum is taken over all cosets $I_{j} \subset G$ (see[4]). Since the topological nature os $G$ at any $x \in G$ is the same as it is at 0 , we choose $x_{0}=0$ in our article.

\section{Main results and proofs}

Define multilinear commutator of fractional integral operator $I_{\alpha}$ as

$$
\left[b, I_{\alpha}\right] f(x)=\int_{G} \prod_{j=1}^{m}\left[b_{j}(x)-b_{j}(y)\right] \frac{f(y)}{|x-y|^{1-\alpha}} d y
$$

and maximal function

$$
f_{\alpha, r}^{*}(x)=\sup _{x \in G_{k}}\left|G_{k}\right|^{\alpha}\left(\frac{1}{\left|G_{k}\right|} \int_{G_{k}}|f(y)|^{r} d y\right)^{1 / r} .
$$

Given any positive integer $m$, for all $1 \leq j \leq m$, we denote by $C_{j}^{m}$ the family of all finite subsets $\sigma=\{\sigma(1), \cdots, \sigma(j)\}$ of $\{1, \cdots, m\}$ of $j$ different elements. For any $\sigma \in C_{j}^{m}$, we associate the complementary sequence $\sigma^{\prime}$ given by $\sigma^{\prime}=\{1,2, \cdots, m\} \backslash \sigma$, and

$\left[b_{\sigma}, I_{\alpha}\right] f(x)=\int_{G}\left[b_{\sigma(1)}(x)-b_{\sigma(1)}(y)\right] \cdots\left[b_{\sigma(j)}(x)-b_{\sigma(j)}(y)\right] \frac{f(y)}{|x-y|^{1-\alpha}} d y$.

suppose $\left\|b_{i}\right\|_{B M O}=1$ for $i=1,2, \cdots, m$. Let $p^{\prime}$ is adjoint index of $p$. We have the following theorem.

Theorem 2.1. Let $b=\left(b_{1}, \cdots, b_{m}\right), b_{i} \in B M O, 1 / q=1 / p-\alpha$, $1<p<1 / \alpha, 0<\alpha<1$, then $\left[b, I_{\alpha}\right]$ maps $L^{p}(G)$ into $L^{q}(G)$.

To prove this theorem, we need the following lemmas.

Lemma 2.1 (see [7]). Let $p>1,1 / q=1 / p-\alpha, 0<\alpha<1$, then

$$
\left\|I_{\alpha} f\right\|_{q} \leq C\|f\|_{p}
$$

Lemma 2.2 (see [5] and [8]). Let $r<p<1 / \alpha, 1 / q=1 / p-\alpha$, $0<\alpha<1$, then

$$
\left\|f_{\alpha, r}^{*}\right\|_{q} \leq C\|f\|_{p}
$$


Lemma 2.3. Let $b=\left(b_{1}, \cdots, b_{m}\right), b_{i} \in B M O, 0<\delta<\epsilon<1$, then there exist a constant $C$ depends on $\delta, \epsilon$, and choose $p_{1}$ that $1<p_{1}<p<$ $1 / \alpha$ such that

$$
\begin{aligned}
& M_{\delta}^{\sharp}\left(\left[b, I_{\alpha}\right] f\right)(x) \\
& \quad \leq C\left\{M_{\epsilon}\left(I_{\alpha} f\right)(x)+\sum_{j=1}^{m-1} \sum_{\sigma \in C_{j}^{m}} M_{\epsilon}\left(\left[b_{\sigma^{\prime}}, I_{\alpha}\right] f\right)(x)+f_{\alpha, p_{1}}^{*}(x)\right\} .
\end{aligned}
$$

Proof. We firstly consider the case of $m=1$, that is,

$$
\begin{aligned}
{\left[b, I_{\alpha}\right] f(x) } & =\int_{G}[b(x)-b(y)] \frac{f(y)}{|x-y|^{1-\alpha}} d y \\
& =[b(x)-\lambda] I_{\alpha} f(x)-I_{\alpha}[(b-\lambda) f](x) .
\end{aligned}
$$

Fix $x$, let $x \in G_{k}$, since

$$
\left.|| \alpha\right|^{\delta}-|\beta|^{\delta}|\leq| \alpha-\left.\beta\right|^{\delta}
$$

for any $\alpha, \beta \in R, 0<\delta<1$, then we have

$$
\begin{aligned}
\left(\left.\frac{1}{\left|G_{k}\right|} \int_{G_{k}}||\left[b, I_{\alpha}\right] f(y)\right|^{\delta}-|c|^{\delta} \mid d y\right)^{1 / \delta} & \\
\leq & \left(\frac{1}{\left|G_{k}\right|} \int_{G_{k}}\left|\left[b, I_{\alpha}\right] f(y)-c\right|^{\delta} d y\right)^{1 / \delta} \\
\leq & C\left(\frac{1}{\left|G_{k}\right|} \int_{G_{k}}\left|[b(y)-\lambda] I_{\alpha} f(y)\right|^{\delta} d y\right)^{1 / \delta} \\
& +C\left(\frac{1}{\left|G_{k}\right|} \int_{G_{k}}\left|I_{\alpha}[(b-\lambda) f](y)-c\right|^{\delta} d y\right)^{1 / \delta} \\
= & \mathrm{I}+\mathrm{II} .
\end{aligned}
$$

choose $\lambda=b_{G_{k}}$ and $1<q<\epsilon / \delta$, using Hölder inequality and Jenson inequality, we can deduce that

$$
\begin{aligned}
I & \leq C\left(\frac{1}{\left|G_{k}\right|} \int_{G_{k}}|b(y)-\lambda|^{\delta q^{\prime}} d y\right)^{1 / \delta q^{\prime}}\left(\frac{1}{\left|G_{k}\right|} \int_{G_{k}}\left|I_{\alpha} f(y)\right|^{\delta q} d y\right)^{1 / \delta q} \\
& \leq C\|b\|_{B M O} M_{\epsilon}\left(I_{\alpha} f\right)(x) .
\end{aligned}
$$

To estimate II, let $f=f_{1}+f_{2}, f_{1}=f \chi_{G_{k}}$, then

$$
\begin{aligned}
\mathrm{II} \leq & C\left(\frac{1}{\left|G_{k}\right|} \int_{G_{k}}\left|I_{\alpha}\left[(b-\lambda) f_{1}\right](y)\right|^{\delta} d y\right)^{1 / \delta} \\
& +\left(\frac{1}{\left|G_{k}\right|} \int_{G_{k}}\left|I_{\alpha}\left[(b-\lambda) f_{2}\right](y)-c\right|^{\delta} d y\right)^{1 / \delta}=\mathrm{III}+\mathrm{IV} .
\end{aligned}
$$


For III, we have

$$
\begin{aligned}
\mathrm{III} & \leq C \frac{1}{\left|G_{k}\right|} \int_{G_{k}}\left|I_{\alpha}\left[(b-\lambda) f_{1}\right](y)\right| d y \\
& \leq C \frac{1}{\left|G_{k}\right|} \int_{G_{k}} \int_{G_{k}}|b(y)-\lambda| \frac{|f(y)|}{|x-y|^{1-\alpha}} d y d x \\
& \leq C \frac{1}{\left|G_{k}\right|^{1-\alpha}} \int_{G_{k}}|b(y)-\lambda||f(y)| d y,
\end{aligned}
$$

choose $p_{1}$ such that $1<p_{1}<p<1 / \alpha$, then

$$
\begin{aligned}
\mathrm{III} & \leq C\left(\frac{1}{\left|G_{k}\right|} \int_{G_{k}}|b(y)-\lambda|^{p_{1}^{\prime}} d y\right)^{1 / p_{1}^{\prime}}\left|G_{k}\right|^{\alpha}\left(\frac{1}{\left|G_{k}\right|} \int_{G_{k}}|f(y)|^{p_{1}} d y\right)^{1 / p_{1}} \\
& \leq C\|b\|_{B M O} f_{\alpha, p_{1}}^{*}(x) .
\end{aligned}
$$

Next, we turn to estimate IV. Let

$$
c=\left(I_{\alpha}\left[(b-\lambda) f_{2}\right]\right)_{G_{k}},
$$

then

$$
\begin{aligned}
\mathrm{IV} \leq & C \frac{1}{\left|G_{k}\right|} \int_{G_{k}}\left|I_{\alpha}\left[(b-\lambda) f_{2}\right](y)-\left(I_{\alpha}\left[(b-\lambda) f_{2}\right]\right)_{G_{k}}\right| d y \\
= & C \frac{1}{\left|G_{k}\right|} \int_{G_{k}} \mid \int_{G \backslash G_{k}} \frac{[b(\omega)-\lambda] f(\omega)}{|y-\omega|^{1-\alpha}} d \omega \\
& -\frac{1}{\left|G_{k}\right|} \int_{G_{k}}\left\{\int_{G \backslash G_{k}} \frac{[b(\omega)-\lambda] f(\omega)}{|z-\omega|^{1-\alpha}} d \omega\right\} d z \mid d y \\
= & C \frac{1}{\left|G_{k}\right|} \int_{G_{k}} \mid \frac{1}{\left|G_{k}\right|} \int_{G_{k}}\left\{\int_{G \backslash G_{k}}[b(\omega)-\lambda] f(\omega)\right. \\
& \times\left[\frac{1}{|y-\omega|^{1-\alpha}}-\frac{1}{\left.\left.|z-\omega|^{1-\alpha}\right] d \omega\right\} d z \mid d y}\right. \\
\leq & C \frac{1}{\left|G_{k}\right|} \int_{G_{k}} \frac{1}{\left|G_{k}\right|} \int_{G_{k}} \sum_{i=-\infty}^{k-1} \int_{G_{i} \backslash G_{i+1}}|b(\omega)-\lambda| \\
& \times|f(\omega)| \frac{|y-z|}{|z-\omega|^{2-\alpha}} d \omega d z d y \\
\leq & C \sum_{i=-\infty}^{k-1} \frac{\left|G_{k}\right|}{\left|G_{i}\right|^{2-\alpha}} \int_{G_{i}}|b(\omega)-\lambda||f(\omega)| d \omega \\
\leq & C \sum_{i=-\infty}^{k-1} \frac{\left|G_{k}\right|}{\left|G_{i}\right|^{1-\alpha}}\left(\frac{1}{\left|G_{i}\right|} \int_{G_{i}}|b(\omega)-\lambda|^{p_{1}^{\prime}} d \omega\right)^{1 / p_{1}^{\prime}} \\
& \times\left(\frac{1}{\left|G_{i}\right|} \int_{G_{i}}^{\left.|f(\omega)|^{p_{1}} d \omega\right)^{1 / p_{1}}}\right.
\end{aligned}
$$




$$
\begin{aligned}
& \leq C \sum_{i=-\infty}^{k-1} \frac{\left|G_{k}\right|}{\left|G_{i}\right|} f_{\alpha, p_{1}}^{*}(x)\left\{\left(\frac{1}{\left|G_{i}\right|} \int_{G_{i}}\left|b(\omega)-b_{G_{i}}\right|^{p_{1}^{\prime}} d \omega\right)^{1 / p_{1}^{\prime}}+\left|b_{G_{i}}-b_{G_{k}}\right|\right\} \\
& \leq C \sum_{i=-\infty}^{k-1} \frac{\left|G_{k}\right|}{\left|G_{i}\right|}\left[1+\frac{1}{2}(k-i)\right] f_{\alpha, p_{1}}^{*}(x)\|b\|_{B M O} \\
& \leq C \sum_{i=-\infty}^{k-1} \frac{m_{i}}{m_{k}}(k-i) f_{\alpha, p_{1}}^{*}(x)\|b\|_{B M O} \\
& \leq C\|b\|_{B M O} f_{\alpha, p_{1}}^{*}(x),
\end{aligned}
$$

where

$$
\begin{aligned}
\left|b_{G_{n}}-b_{G_{n+1}}\right| & \leq \frac{1}{\left|G_{n}\right|} \int_{G_{n}}\left|b-b_{G_{n+1}}\right| d x \\
& \leq \frac{\left|G_{n+1}\right|}{\left|G_{n}\right|} \frac{1}{\left|G_{n+1}\right|} \int_{G_{n+1}}\left|b(x)-b_{G_{n+1}}\right| d x \\
& \leq \frac{\left|G_{n+1}\right|}{\left|G_{n}\right|}\|b\|_{B M O} \\
& \leq \frac{1}{2}\|b\|_{B M O},
\end{aligned}
$$

for any $n \in \mathbb{Z}$, and

$$
\begin{aligned}
\left|b_{G_{i}}-b_{G_{k}}\right| & =\left|b_{G_{i}}-b_{G_{i+1}}\right|+\left|b_{G_{i+1}}-b_{G_{i+2}}\right|+\cdots+\left|b_{G_{k-1}}-b_{G_{k}}\right| \\
& =\frac{1}{2}(k-i)\|b\|_{B M O} .
\end{aligned}
$$

Therefore, we have proved that

$$
M_{\delta}^{\sharp}\left(\left[b, I_{\alpha}\right] f\right)(x) \leq C\|b\|_{B M O}\left[M_{\epsilon}\left(I_{\alpha} f\right)(x)+f_{\alpha, p_{1}}^{*}(x)\right]
$$

for $m=1$.

If $m \geq 2$, let $\lambda=\left(\lambda_{1}, \cdots, \lambda_{m}\right)$, we have

$$
\begin{aligned}
{\left[b, I_{\alpha}\right] f(x)=} & \int_{G} \prod_{j=1}^{m}\left[\left(b_{j}(x)-b_{j}(y)\right] \frac{f(y)}{|x-y|^{1-\alpha}} d y\right. \\
= & \int_{G} \prod_{j=1}^{m}\left[\left(b_{j}(x)-\lambda_{j}+\lambda_{j}-b_{j}(y)\right] \frac{f(y)}{|x-y|^{1-\alpha}} d y\right. \\
= & \prod_{j=1}^{m}\left[\left(b_{j}(x)-\lambda_{j}\right] I_{\alpha} f(x)+(-1)^{m} I_{\alpha}\left[\prod_{j=1}^{m}\left(b_{j}(x)-\lambda_{j}\right) f\right](x)\right. \\
& +\sum_{j=1}^{m-1} \sum_{\sigma \in C_{j}^{m}} C_{m, j}[b(x)-\lambda]_{\sigma}\left[b_{\sigma^{\prime}}, I_{\alpha}\right](f)(x),
\end{aligned}
$$


where $C_{m, j}$ are constants depending on $m$ and $j$.

Now, for fixed $x \in G$, for any number $c$ and $G_{k} \ni x$, since $0<\delta<1$, we have

$$
\begin{aligned}
\left(\left.\frac{1}{\left|G_{k}\right|} \int_{G_{k}}||\left[b, I_{\alpha}\right] f(y)\right|^{\delta}-|c|^{\delta} \mid d y\right)^{1 / \delta} \\
\leq\left(\frac{1}{\left|G_{k}\right|} \int_{G_{k}}\left|\left[b, I_{\alpha}\right] f(y)-c\right|^{\delta} d y\right)^{1 / \delta} \\
\leq C\left(\frac{1}{\left|G_{k}\right|} \int_{G_{k}}\left|\prod_{j=1}^{m}\left[b_{j}(y)-\lambda_{j}\right] I_{\alpha}(f)(y)\right|^{\delta} d y\right)^{1 / \delta} \\
\quad+\sum_{j=1}^{m-1} \sum_{\sigma \in C_{j}^{m}}\left(\frac{1}{\left|G_{k}\right|} \int_{G_{k}}\left|[b(y)-\lambda]_{\sigma}\left[b_{\sigma^{\prime}}, I_{\alpha}\right](f)(y)\right|^{\delta} d y\right)^{1 / \delta} \\
\quad+C\left(\frac{1}{\left|G_{k}\right|} \int_{G_{k}}\left|I_{\alpha}\left[\prod_{j=1}^{m}\left(b_{j}-\lambda_{j}\right) f\right](y)-c\right|^{\delta} d y\right)^{1 / \delta} \\
=\mathrm{I}+\mathrm{II}+\mathrm{III} .
\end{aligned}
$$

For the first two parts I and II, choose $\lambda_{j}=\left(b_{j}\right)_{G_{k}}, j=1,2 \cdots, m$, and $1<q<\delta / \epsilon$, similar to the case of $m=1$, we can deduce that

$$
\mathrm{I} \leq C M_{\epsilon}\left(I_{\alpha} f\right)(x),
$$

and

$$
\mathrm{II} \leq C \sum_{j=1}^{m-1} \sum_{\sigma \in C_{j}^{m}} M_{\epsilon}\left(\left[b_{\sigma^{\prime}}, I_{\alpha}\right] f\right)(x) .
$$

For III, let $f=f_{1}+f_{2}, f_{1}=f \chi_{G_{k}}$, then

$$
\begin{aligned}
\mathrm{III} \leq & C\left\{\frac{1}{\left|G_{k}\right|} \int_{G_{k}}\left|I_{\alpha}\left[\prod_{j=1}^{m}\left(b_{j}-\lambda_{j}\right) f_{1}\right](y)\right|^{\delta} d y\right\}^{1 / \delta} \\
& +C\left\{\frac{1}{\left|G_{k}\right|} \int_{G_{k}}\left|I_{\alpha}\left[\prod_{j=1}^{m}\left(b_{j}-\lambda_{j}\right) f_{2}\right](y)-c\right|^{\delta} d y\right\}^{1 / \delta} \\
= & \mathrm{IV}+\mathrm{V} .
\end{aligned}
$$

By Jenson inequality, we have

$$
\begin{aligned}
\mathrm{III} & \leq C \frac{1}{\left|G_{k}\right|} \int_{G_{k}}\left|I_{\alpha}\left[\prod_{j=1}^{m}\left(b_{j}-\lambda_{j}\right) f_{1}\right](y)\right| d y \\
& \leq C \frac{1}{\left|G_{k}\right|} \int_{G_{k}} \int_{G_{k}} \prod_{j=1}^{m}\left|b_{j}(z)-\lambda_{j}\right| \frac{|f(z)|}{|y-z|^{1-\alpha}} d z d y
\end{aligned}
$$




$$
\begin{aligned}
& \leq C \frac{1}{\left|G_{k}\right|} \int_{G_{k}} \prod_{j=1}^{m}\left|b_{j}(z)-\lambda_{j}\right||f(z)|\left(\int_{G_{k}} \frac{1}{|y-z|^{1-\alpha}} d y\right) d z \\
& \leq C\left(\frac{1}{\left|G_{k}\right|} \int_{G_{k}}\left|\prod_{j=1}^{m}\left(b_{j}(z)-\lambda_{j}\right)\right|^{p_{1}^{\prime}} d z\right)^{1 / p_{1}^{\prime}}\left|G_{k}\right|^{\alpha}\left(\frac{1}{\left|G_{k}\right|} \int_{G_{k}}|f(z)|^{p_{1}} d z\right)^{1 / p_{1}} \\
& \leq C \prod_{j=1}^{m}\left(\frac{1}{\left|G_{k}\right|} \int_{G_{k}}\left|b_{j}(z)-\lambda_{j}\right|^{m p_{1}^{\prime}} d z\right)^{1 / m p_{1}^{\prime}} f_{\alpha, p_{1}}^{*}(x) \\
& \leq C\|b\|_{B M O} f_{\alpha, p_{1}}^{*}(x) .
\end{aligned}
$$

To estimate $V$, let

$$
c=\left(I_{\alpha}\left[\prod_{j=1}^{m}\left(b_{j}-\lambda_{j}\right) f_{2}\right]\right)_{G_{k}},
$$

similar to the case in $m=1$, it can follow that

$$
\begin{aligned}
\mathrm{IV} \leq & C \frac{1}{\left|G_{k}\right|} \int_{G_{k}}\left|I_{\alpha}\left[\prod_{j=1}^{m}\left(b_{j}-\lambda_{j}\right) f_{2}\right](y)-\left[I_{\alpha}\left(\prod_{j=1}^{m}\left(b_{j}-\lambda_{j}\right) f_{2}\right)\right]_{G_{k}}\right| d y \\
\leq & C \sum_{i=-\infty}^{k-1} \frac{\left|G_{k}\right|}{\left|G_{i}\right|^{2-\alpha}} \int_{G_{i}} \prod_{j=1}^{m}\left|b_{j}(\omega)-\lambda_{j}\right||f(\omega)| d \omega \\
\leq & C \sum_{i=-\infty}^{k-1} \frac{\left|G_{k}\right|}{\left|G_{i}\right|^{1-\alpha}}\left(\frac{1}{\left|G_{i}\right|} \int_{G_{i}}\left|\prod_{j=1}^{m}\left(b_{j}(\omega)-\lambda_{j}\right)\right|^{p_{1}^{\prime}} d \omega\right)^{1 / p_{1}^{\prime}} \\
& \times\left(\frac{1}{\left|G_{i}\right|} \int_{G_{i}}|f(\omega)|^{p_{1}} d \omega\right)^{1 / p_{1}} \\
\leq & C \sum_{i=-\infty}^{k-1} \frac{\left|G_{k}\right|}{\left|G_{i}\right|} f_{\alpha, p_{1}}^{*}(x) \times \prod_{j=1}^{m}\left[\left(\frac{1}{\left|G_{i}\right|} \int_{G_{i}}\left|b_{j}(\omega)-\left(b_{j}\right)_{G_{i}}\right|^{m p_{1}^{\prime}} d \omega\right)^{1 / m p_{1}^{\prime}}\right. \\
& \left.+\left|\left(b_{j}\right)_{G_{i}}-\left(b_{j}\right)_{G_{k}}\right|\right] \\
\leq & C \sum_{i=-\infty}^{k-1} \frac{m_{i}}{m_{k}}(k-i)^{m} f_{\alpha, p_{1}}^{*}(x)\|b\|_{B M O} \\
\leq & C\|b\|_{B M O} f_{\alpha, p_{1}}^{*}(x) .
\end{aligned}
$$

Combine with I, II, IV, V, we finish the proof of Lemma 2.3 .

Proof of Theorem 2.1. We first take it for granted that $M\left(\left[b, I_{\alpha}\right] f\right) \in$ $L^{q}(G)$ and we'll check this to the end of the proof. 
We proceed by induction on $m$. For $m=1$, by lemma 2.1, 2.2 and 2.3, we have

$$
\begin{aligned}
\left\|\left[b, I_{\alpha}\right] f\right\|_{q} & \leq C\left\|M_{\delta}^{\sharp}\left(\left[b, I_{\alpha}\right] f\right)\right\|_{q} \\
& \leq C\left\|M_{\epsilon}\left(I_{\alpha} f\right)\right\|_{q}+C\left\|f_{\alpha, p_{1}}^{*}\right\|_{q} \\
& \leq C\left\|I_{\alpha} f\right\|_{q}+C\|f\|_{p} \\
& \leq C\|f\|_{p} .
\end{aligned}
$$

Suppose now that for $m-1$ the theorem is true, and let us prove it for $m$. The same argument as used above and the induction hypothesis give

$$
\begin{aligned}
\left\|\left[b, I_{\alpha}\right] f\right\|_{q} & \leq C \|\left(M_{\epsilon}\left(I_{\alpha} f\right)\left\|_{q}+C \sum_{j=1}^{m-1} \sum_{\sigma \in C_{j}^{m}}\right\| M_{\epsilon}\left(\left[b_{\sigma^{\prime}}, I_{\alpha}\right] f\right)\left\|_{q}+C\right\| f \|_{p}\right. \\
& \leq C\|f\|_{p} .
\end{aligned}
$$

Now let us check that $M\left(\left[b, I_{\alpha}\right] f\right) \in L^{q}(G)$. By the boundedness of maximal operator, we only need to prove $\left[b, I_{\alpha}\right] f \in L^{q}(G)$. Suppose for any $j(j=1,2 \cdots, m), b_{j}$ is bounded, since $C_{c}^{\infty}(G)$ is dense in $L^{p}(G)(p>1)$, we only need to consider the function $f$ with compact support. Suppose $\operatorname{supp} f \subset G_{k}$,we have

$$
\begin{aligned}
\left\|\left[b, I_{\alpha}\right] f\right\|_{q} & =\left(\int_{G_{k}}\left|\left[b, I_{\alpha}\right](f)(x)\right|^{q} d x\right)^{1 / q}+\left(\int_{G \backslash G_{k}}\left|\left[b, I_{\alpha}\right](f)(x)\right|^{q} d x\right)^{1 / q} \\
& =\mathrm{A}_{1}+\mathrm{A}_{2} .
\end{aligned}
$$

Using the boundedness of $b_{i}$, we get

$$
\begin{aligned}
\mathrm{A}_{1} & \leq C\left(\int_{G_{k}}\left|\int_{G}[b(x)-b(y)] \frac{f(y)}{|x-y|^{1-\alpha}} d y\right|^{q} d x\right)^{1 / q} \\
& \leq C\left(\int_{G_{k}}\left|I_{\alpha}(f)(x)\right|^{q} d x\right)^{1 / q} \\
& \leq C\|f\|_{p} .
\end{aligned}
$$

For the second term, according to the boundedness of $b_{i}$ and $x \notin G_{k}$, $y \in G_{k},|x-y| \sim|x|$, we have

$$
\begin{aligned}
\left|\left[b, I_{\alpha}\right] f\right| & \leq C \int_{G_{k}} \prod_{j=1}^{m}\left|b_{j}(x)-b_{j}(y)\right| \frac{|f(y)|}{|x-y|^{1-\alpha}} d y \\
& \leq C \int_{G_{k}} \frac{|f(y)|}{|x-y|^{1-\alpha}} d y
\end{aligned}
$$




$$
\begin{aligned}
& \leq C \frac{1}{|x|^{1-\alpha}} \int_{G_{k}}|f(y)| d y \\
& \leq C\left|G_{k}\right|^{\alpha}\left(\frac{1}{\left|G_{k}\right|} \int_{G_{k}}|f(y)|^{p_{1}} d y\right)^{1 / p_{1}} \\
& \leq C f_{\alpha, p_{1}}^{*}(x),
\end{aligned}
$$

Thus,

$$
\mathrm{A}_{2} \leq C\left(\int_{G \backslash G_{k}}\left|f_{\alpha, p_{1}}^{*}(x)\right|^{q} d x\right)^{1 / q} \leq C\left\|f_{\alpha, p_{1}}^{*}\right\|_{q},
$$

which is finite by lemma 2.2 .

For the general case, we will truncate the symbols $b_{j}$ as follows. Denote $b_{j}^{N}=\min \left\{b_{j}, N\right\}$, take into account the fact that $f$ has compact support, we deduce that any product $b_{j_{1}}^{N} \cdots b_{j_{i}}^{N} f$ converges in any $L^{q}(G)(q>1)$ to $b_{j_{1}} \cdots b_{j_{i}} f$ as $N \rightarrow \infty$. By the above discussion, we have

$$
\left\|\left[b^{N}, I_{\alpha}\right](f)\right\|_{q} \leq C\left\|b^{N}\right\|_{B M O} \|\left. f\right|_{p} .
$$

By Fatou's lemma, we conclude that the theorems holds for this general case. The theorem is proved.

Furthermore, we can discuss the boundedness on Hardy spaces.

Definition 2.1. Let $0<p \leq 1 \leq q \leq \infty, p \neq q$, a nonnegative integer $s \geq[1 / p-1], b=\left(b_{1}, \cdots, b_{m}\right), b_{i} \in B M O(G), i=1,2, \cdots, m$. A function $a(x) \in L_{l o c}^{q}(G)$ is said to be a $(p, q, b)$-atom if it satisfies:

(i) for some $k \in \mathrm{Z}$, supp $a \subset G_{k}$;

(ii) $\|a\|_{q} \leq\left|G_{k}\right|^{1 / q-1 / p}$;

(iii) $\int_{G_{k}} a(y) d y=\int_{G_{k}} a(y) \Pi_{l \in \sigma} b_{l}(y) d y=0$, for any $\sigma \in C_{j}^{m}, 1 \leq j \leq$ $m$.

Definition 2.2. The Hardy spaces $H_{b}^{p}(G)$ are defined by

$$
\begin{aligned}
H_{b}^{p}(G)=\left\{f: f \in S^{\prime}(G), f=\sum_{k=-\infty}^{\infty} \lambda_{k} a_{k}, a_{k} \text { is }(p, q, b)-\text { atom },\right. \\
\\
\left.\sum_{k=-\infty}^{\infty}\left|\lambda_{k}\right|^{p}<\infty\right\} .
\end{aligned}
$$

and

$$
\|f\|_{H_{b}^{p}(G)} \sim \inf \left\{\left(\sum_{k=-\infty}^{\infty}\left|\lambda_{k}\right|^{p}\right)^{1 / p}\right\},
$$

where the infimum is taken over all the decomposition of $f$ as above. 
Theorem 2.2. Let $b$ is the same as Theorem 2.1. Let $1 / 2<p \leq 1$, $1 / q=1 / p-\alpha, 0<\alpha<1$, then $\left[b, I_{\alpha}\right]$ is a bounded operator from $H_{b}^{p}(G)$ into $L^{q}(G)$.

Proof. We only need to prove theorem for the $(p, \infty, b)$-atom. Suppose supp $a \subset G_{k}$, then

$\left\|\left[b, I_{\alpha}\right] a\right\|_{q} \leq\left(\int_{G_{k}}\left|\left[b, I_{\alpha}\right] a(x)\right|^{q} d x\right)^{1 / q}+\left(\int_{G \backslash G_{k}}\left|\left[b, I_{\alpha}\right] a(x)\right|^{q} d x\right)^{1 / q}=\mathrm{I}+\mathrm{II}$.

Choose $p_{1}, q_{1}$ such that $1<p_{1}<q_{1}<\infty$ and $1 / q=1 / p-\alpha$, then $q<q_{1}$. By Hölder inequality and Theorem 2.1, we have

$$
\begin{aligned}
\mathrm{I} & \leq C\left(\int_{G_{k}}\left|\left[b, I_{\alpha}\right] a(x)\right|^{q_{1}} d x\right)^{1 / q_{1}}\left|G_{k}\right|^{1 / q-1 / q_{1}} \\
& \leq C\|a\|_{p_{1}}\left|G_{k}\right|^{1 / q-1 / q_{1}} \\
& \leq C\|a\|_{\infty}\left|G_{k}\right|^{1 / p_{1}+1 / q-1 / q_{1}} \\
& \leq C\left|G_{k}\right|^{1 / p_{1}-1 / p+1 / q-1 / q_{1}} \\
& =C
\end{aligned}
$$

Note that $x \in G \backslash G_{k}$, and $y \in G_{k}$, let $\lambda_{j}=\left(b_{j}\right)_{G_{k}}$ for any $j$, then

$$
\begin{aligned}
\left|\left[b, I_{\alpha}\right] a(x)\right| & =\left|\int_{G_{k}} \prod_{j=1}^{m}\left[b_{j}(x)-b_{j}(y)\right] \frac{a(y)}{|x-y|^{1-\alpha}} d y\right| \\
& =\left|\sum_{j=0}^{m} \sum_{\sigma \in C_{j}^{m}} C[b(x)-\lambda]_{\sigma} \int_{G_{k}}[b(x)-\lambda]_{\sigma^{\prime}} \frac{a(y)}{|x-y|^{1-\alpha}} d y\right|
\end{aligned}
$$

Using the vanishing moment condition (iii), we get

$$
\begin{aligned}
\left|\left[b, I_{\alpha}\right] a(x)\right|= & \mid \sum_{j=0}^{m} \sum_{\sigma \in C_{j}^{m}} C[b(x)-\lambda]_{\sigma} \int_{G_{k}}[b(x)-\lambda]_{\sigma^{\prime}} a(y) \\
& \times\left[\frac{1}{|x-y|^{1-\alpha}}-\frac{1}{|x|^{1-\alpha}}\right] d y \mid \\
\leq & C \sum_{j=0}^{m} \sum_{\sigma \in C_{j}^{m}}\left|[b(x)-\lambda]_{\sigma}\right|\left(\frac{\left|G_{k}\right|}{|x|^{2-\alpha}} \int_{G_{k}}\left|[b(x)-\lambda]_{\sigma^{\prime}} a(y)\right| d y\right) .
\end{aligned}
$$


Similar to the proof of Theorem 2.1, we deduce that

$$
\begin{aligned}
\mathrm{II} \leq & C \sum_{j=0}^{m} \sum_{\sigma \in C_{j}^{m}} \int_{G_{k}}\left|[b(x)-\lambda]_{\sigma^{\prime}} a(y)\right| d y \\
& \times\left[\sum_{i=-\infty}^{k-1} \int_{G_{i} \backslash G_{i+1}}\left|[b(x)-\lambda]_{\sigma}\right|^{q}\left\{\frac{\left|G_{k}\right|}{|x|^{2-\alpha}}\right\}^{q} d x\right]^{1 / q} \\
\leq & C \sum_{j=0}^{m} \sum_{\sigma \in C_{j}^{m}} \int_{G_{k}}\left|[b(x)-\lambda]_{\sigma^{\prime}} a(y)\right| d y \\
& \times \sum_{i=-\infty}^{k-1} \frac{\left|G_{k}\right|}{\left|G_{i}\right|^{2-\alpha}}\left(\int_{G_{i} \backslash G_{i+1}}\left|[b(x)-\lambda]_{\sigma}\right|^{q} d x\right)^{1 / q} \\
\leq & C\|a\|_{\infty} \sum_{j=0}^{m} \sum_{\sigma \in C_{j}^{m}}\left|G_{k}\right|\left(\frac{1}{\left|G_{k}\right|} \int_{G_{k}}\left|[b(x)-\lambda]_{\sigma^{\prime}}\right| d y\right) \\
& \times \sum_{i=-\infty}^{k-1} \frac{\left|G_{k}\right|}{\left|G_{i}\right|^{2-\alpha}}\left|G_{i}\right|^{1 / q}\left(\frac{1}{\left|G_{i}\right|} \int_{G_{i}}\left|[b(x)-\lambda]_{\sigma}\right|^{q} d x\right)^{1 / q} \\
\leq & C\|a\|_{\infty}\left|G_{k}\right|\|b\|_{B M O} \sum_{i=-\infty}^{k-1} \frac{\left|G_{k}\right|}{\left|G_{i}\right|^{2-\alpha-1 / q}}(k-i)^{m} \\
\leq & C\|b\|_{B M O} \sum_{i=-\infty}^{k-1} \frac{\left|G_{k}\right|^{2-1 / p}}{\left|G_{i}\right|^{2-\alpha-1 / q}}(k-i)^{m} \\
\leq & C\|b\|_{B M O},
\end{aligned}
$$

Here $m \geq 1,1 / 2<p \leq 1$ and $1 / q=1 / p-\alpha$.

Therefore, Theorem 2.2 is proved.

\section{References}

[1] C. Perez, Sharp estimates for commutators of singular integrals via interations of the Hardy-littlewood maximal function, J. Fourier Anal. Appl., 3 (1997), 743-756.

[2] C. Perez and R. Trujillo-Gonazalez, Sharp weighted estimates for multilinear commutators, J. London Math. Soc., 65 (2002), 672-692.

[3] R. R. Coifman, R. Rochberg and G. Weiss, Factorization theorems for Hardy spaces in several variables, Ann. of Math., 103 (1976), 611-635.

[4] J. E. Daly and D. S. Kurtz, BMO and singular integrals over local fileds, J. Austral. Math. Soc. (Series A.), 54 (1993), 321-333. 
[5] T. Guo and Y. Wang, The boundedness of fractional integral operators on Morrey spaces in Vilenkin groups, Math. in Practice and Theory, 35 (8) (2005), 221-223.

[6] S. Janson, Mean oscillation and commutators of singular integral operators, Ark. Math., 16 (1978), 263-270.

[7] S. Lan, Boundedness of fractional integral operators in Herz-type spaces on locally compact Vilenkin groups, Approx. Theory \& its Appl., 18 (2002), 15-25.

[8] T. S. Quek and D. Yang, Generalized Calderón-Zygmund operators on bounded locally compact Vilenkin group, Advance in Math., 30 (6) (2001), 515-524.

[9] A. Uchiyama, On the compactness of operators of Hankel type, Tôhoku Math. J., 30 (1978), 163-171.

[10] W. Zhou, The boundedness of mutilinear commutators of singular integral and fractional integral, Thesis, Hunan University, 2003.

Department of Mathematics

Dalian Maritime University

Dalian, Liaoning

P. R. China, 116026

(E-mail : tangcq2000@yahoo.com.cn)

(Received : November 2005) 


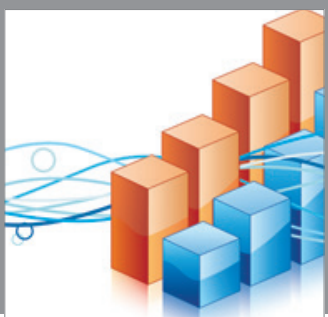

Advances in

Operations Research

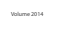

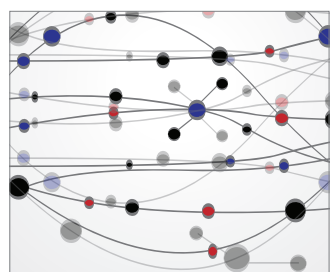

\section{The Scientific} World Journal
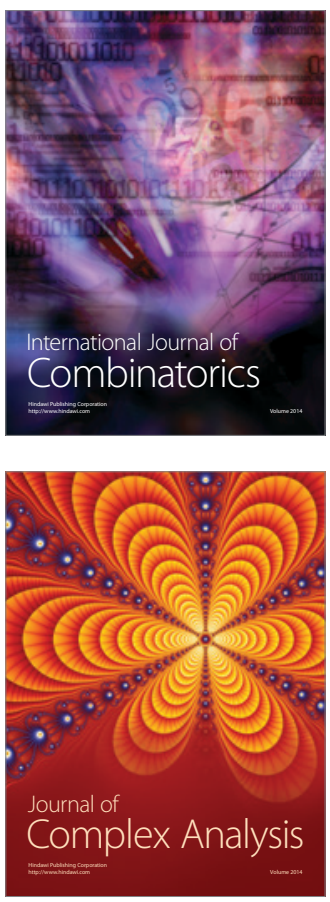

International Journal of

Mathematics and

Mathematical

Sciences
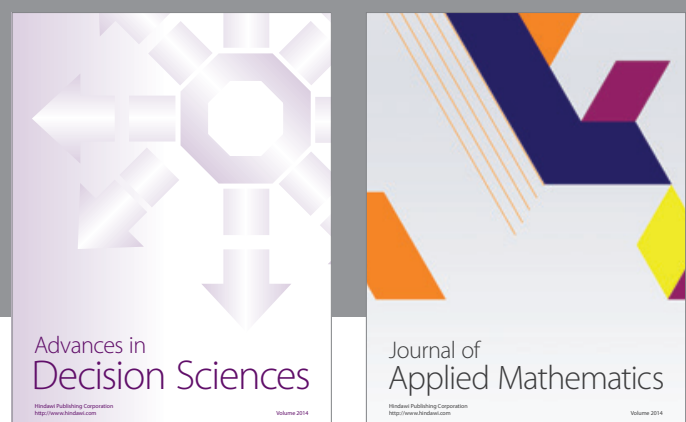

Journal of

Applied Mathematics
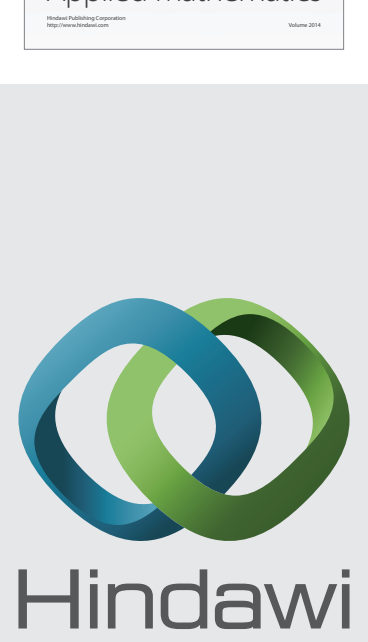

Submit your manuscripts at http://www.hindawi.com
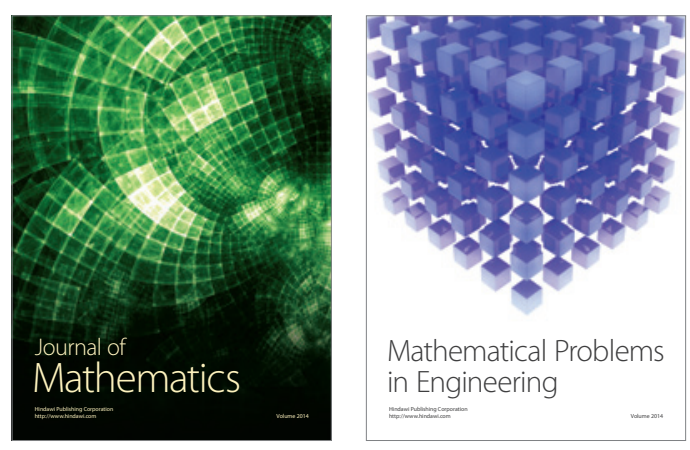

Mathematical Problems in Engineering
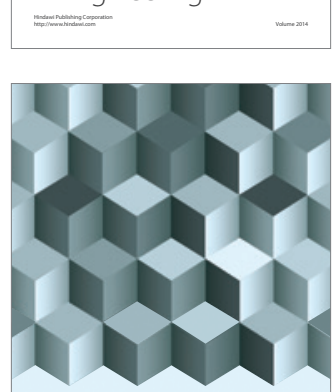

Journal of

Function Spaces
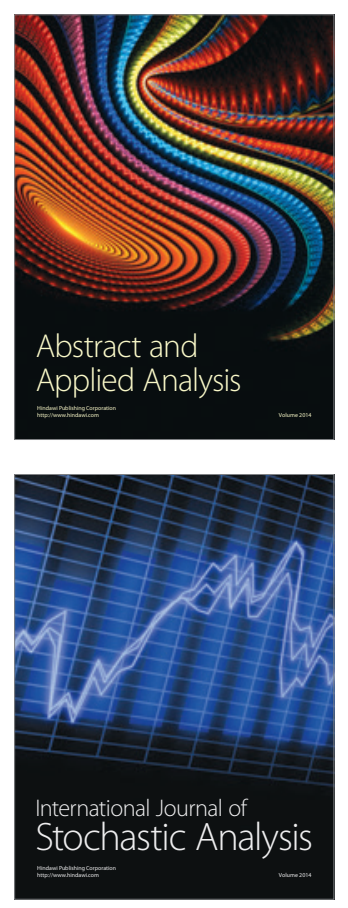

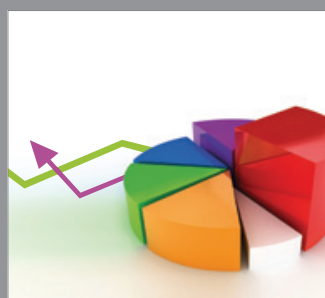

ournal of

Probability and Statistics

Promensencen
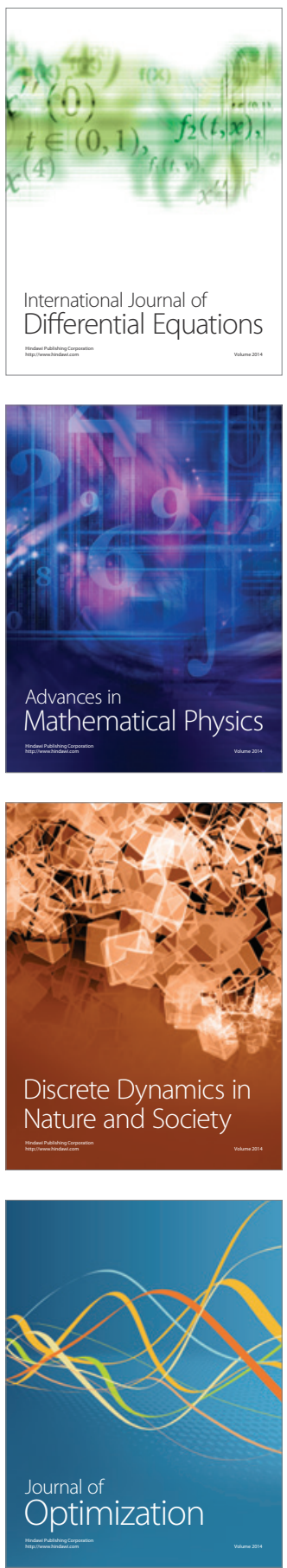